\title{
Impacto de um programa de intervenção sobre o estilo de vida nos perfis metabólico, antropométrico e dietético em nipo-brasileiros com e sem síndrome metabólica
}

\author{
Impact of a lifestyle intervention program on metabolic, \\ anthropometrical and dietary profile of Japanese- \\ Brazilians with and without metabolic syndrome
}

Renata Damião', Daniela Saes Sartorelli², Amélia Hirai Marselle Rodrigues Bevilacqua ${ }^{3}$, Vera Lúcia Morais Antonio de Salvo3, Sandra Roberta Gouvea Ferreira ${ }^{4}$, Suely Godoy Agostinho Gimeno ${ }^{3}$, Grupo de Estudos em Diabetes na População Nipo-Brasileira ${ }^{5}$

\footnotetext{
'Departamento de Nutrição, Universidade Federal do Triângulo Mineiro (UFTM), Uberaba, MG, Brasil ${ }^{2}$ Departamento de Medicina Social, Faculdade de Medicina de Ribeirão Preto, Universidade de São Paulo (FMRP-USP), Ribeirão Preto, SP, Brasil ${ }^{3}$ Departamento de Medicina Preventiva, Universidade Federal de São Paulo (Unifesp), São Paulo, SP, Brasil

${ }^{4}$ Departamento de Nutrição, Faculdade de Saúde Pública, USP, São Paulo, SP, Brasil ${ }^{5}$ Apêndice
}

\section{RESUMO}

Objetivo: Avaliar as mudanças nos perfis metabólico e nutricional de nipo-brasileiros com e sem síndrome metabólica, após dois anos de participação em programa de intervenção. Materiais e métodos: Trata-se de estudo experimental não controlado. A pesquisa incluiu três momentos de avaliação clínico-laboratorial: basal (2005), após o primeiro ano de intervenção (2006) e ao final do segundo ano (2007). Na presente análise, utilizaram-se informações de 360 indivíduos examinados nos três momentos. Resultados: Ambos os grupos apresentaram melhora no perfil metabólico e antropométrico, após o segundo ano de intervenção. Observaram-se diminuição na ingestão de gorduras totais, ácidos graxos saturados, colesterol e aumento de fibras totais nos homens sem síndrome metabólica. No sexo feminino sem síndrome metabólica, observou-se redução de gordura total $(p=0,003)$ e ácido graxo monoinsaturado $(p=0,002)$. Conclusão: Os resultados encontrados mostraram impacto positivo do programa de intervenção nos perfis metabólico e nutricional dos nipo-brasileiros com e sem síndrome metabólica. Arq Bras Endocrinol Metab. 2011;55(2):134-45

\section{Descritores}

Epidemiologia nutricional; intervenção nutricional; síndrome metabólica; nipo-brasileiros; nutrição; doenças crônicas não transmissiveis

\section{ABSTRACT}

Correspondência para: Renata Damião Universidade Federal do Triângulo Mineiro,

Departamento de Nutrição Av. Getúlio Guarita, 159 38025-440 - Uberaba, MG, Brasil damiaorenata@hotmail.com

Objective: We evaluated the changes in biochemical and nutritional profiles of Japanese-Brazilians with and without metabolic syndrome after two years of participation in the intervention program. Materials and methods: It was a non-controlled experimental study. The biochemical and clinical assessments were conducted at baseline (2005), after a year (2006) and after two years (2007) of intervention. On the present study, data of 360 individuals, who participated on the three assessments were considered. Results: Both groups presented improvements on the anthropometric and metabolic profile, after two years of intervention. It was observed reduction in the intake of total fat, saturated fat, and dietary cholesterol, and increased intake of fiber among men without metabolic syndrome. It was observed reduction in the intake of total fat $(p=0,003)$ and monounsaturated fatty acid $(p=0,002)$. Conclusion:The results showed a positive impact of the lifestyle intervention program in metabolic and nutritional profile of Japanese-Brazilians with and without metabolic syndrome. Arq Bras Endocrinol Metab. 2011;55(2):134-45

\section{Keywords}

Nutritional epidemiology; nutrition intervention; metabolic syndrome; Japanese-Brazilian; nutrition; chronic diseases 


\section{INTRODUÇÃO}

$\mathrm{A}$ síndrome metabólica (SM) caracterizada pela intolerância à glicose, hipertensão arterial, dislipidemia e adiposidade corporal foi inicialmente descrita por Reaven (1988) (1) e associa-se ao maior risco de ocorrência de diabetes mellitus tipo 2 (DM2) e doença cardiovascular (2). Sua origem envolve fatores genéticos e ambientais. Enquanto estudos de famílias mostraram haver agregação familiar de casos de resistência à insulina, outros reforçam o papel do ganho de peso e sedentarismo para deterioração da sensibilidade tecidual a esse hormônio (3).

A literatura dispõe hoje de estudos epidemiológicos, realizados principalmente em países desenvolvidos, que fornecem evidências inequívocas da eficácia das mudanças no estilo de vida, na incidência do DM2 $(4,5)$. Resultados de estudos conduzidos em indivíduos portadores de tolerância à glicose diminuída e excesso de peso mostraram a superioridade das mudanças no estilo de vida sobre a intervenção medicamentosa (6).

Estudos comunitários também sugerem benefícios de medidas de intervenção na prevenção do DM2. No entanto, a maioria das pesquisas nessa linha apresenta limitações quanto a seu desenho, incluindo baixa porcentagem de adesão, falta de informação sobre as perdas de seguimento ou curto período de intervenção, comprometendo a avaliação da eficácia desses programas (7).

As contribuições de fatores genéticos e ambientais para a elevada prevalência de DM2 na população nipo-brasileira é incontestável (8). A prevenção ou retardo do desenvolvimento da doença entre esses indivíduos pode reverter em melhoria significativa na qualidade de vida e, consequentemente, na redução de custos em saúde pública com as complicações crônicas da doença. É bem conhecido que o tratamento do DM2 é complexo e dispendioso. O controle metabólico inadequado predispõe a incapacitações físicas dos sujeitos (cegueira, insuficiência renal, amputações, entre outros), a elevados custos sociais e financeiros e à mortalidade prematura. Assim, dentro desse contexto, o presente estudo teve como objetivo avaliar as mudanças nos perfis metabólico e nutricional de nipo-brasileiros com e sem síndrome metabólica, após dois anos de participação em programa de intervenção sobre o estilo de vida (2005-2007).

\section{SUJEITOS E MÉTODOS}

O presente trabalho utilizou os dados da terceira fase do estudo realizado pelo Japanese-Brazilians Diabetes Study Group (JBDSG). Desde 1993, o estudo "Diabe- tes mellitus e doenças associadas na população nipo-brasileira de Bauru" vem sendo conduzido com o objetivo de avaliar aspectos epidemiológicos e clínicos do DM2 e doenças associadas entre os migrantes japoneses e seus descendentes no Brasil (8).

Considerando o perfil cardiovascular desfavorável entre os nipo-brasileiros, descrito pelo JBDSG em pesquisa anterior (9), caracterizado por alta porcentagem de intolerância à glicose $(70 \%)$, hipertensão $(58 \%)$, dislipidemia $(65 \%)$ e obesidade central $(57 \%)$, todos os participantes avaliados em $2000(\mathrm{n}=1.330)$ e também aqueles que completaram 30 anos de idade, no início do presente estudo ("novos"), foram convidados a participar do programa de intervenção sobre o estilo de vida.

Tal programa foi planejado com o objetivo de estimular mudanças no estilo de vida desses nipo-brasileiros, com ênfase nos hábitos alimentares, visando reduzir o risco cardiovascular desses indivíduos. Tratou-se de estudo do tipo experimental não controlado. Resultados preliminares para o sexo feminino foram previamente publicados (10).

Participaram do programa de intervenção 736 nipo-brasileiros de ambos os sexos, de primeira (nascidos no Japão ou isseis) e segunda geração (nascidos no Brasil ou nisseis). Para o presente estudo, excluíram-se 161 (21,9\%) indivíduos que participaram do programa em apenas um único momento, 197 (26,7\%) examinados em duas ocasiões e 18 indivíduos sem dados completos, no início da pesquisa, sobre a ingestão dietética ou perfil bioquímico. Esses indivíduos eram semelhantes aos com três avaliações segundo sexo, idade, geração, IMC e circunferência da cintura $(\mathrm{p}>0,05)$. Utilizaram-se para a presente análise informações de 360 indivíduos (48,9\%) avaliados no início do estudo (basal, em 2005), após o primeiro ano de intervenção (2006) e ao final do segundo ano (2007).

A divulgação do programa de intervenção se deu mediante cartas e eventos realizados na comunidade. Todos os voluntários foram informados dos objetivos do estudo, dos riscos mínimos associados à coleta de sangue com material descartável e das vantagens de participação do programa de estilo de vida saudável, assegurando a confiabilidade e sigilo das informações. A concordância em participar ocorreu mediante assinatura em termo de consentimento livre e esclarecido. O presente artigo foi desenvolvido de acordo com os princípios étnicos contidos na Declaração de Helsinki (1964, reformulada em 1975, 1983, 1989, $1996 \mathrm{e}$ 2000) da World Medical Association e foi aprovado pelo Comitê de Ética em Pesquisa da Universidade Federal de São Paulo (Unifesp), sob protocolo número 1249/06. 


\section{MEDIDAS DE AVALIAÇÃO}

Nos três momentos de avaliação, convidaram-se todos os indivíduos a comparecer em jejum (mínimo de 10 horas) ao laboratório de análises clínicas da Universidade Sagrado Coração de Jesus (Bauru), onde ocorreram a avaliação bioquímica e o exame físico (equipe médica da Unifesp). O exame físico incluiu a coleta de medidas antropométricas, de pressão arterial e avaliação das práticas atuais de atividade física.

Obtiveram-se as medidas de peso (em $\mathrm{kg}$ ) e altura (em metros) com balança digital (capacidade de $150 \mathrm{~kg}$, precisão de $100 \mathrm{~g}$ ) e estadiômetro portátil, respectivamente. Essas medidas foram realizadas com o indivíduo utilizando roupas leves e sem sapatos. Obteve-se a circunferência da cintura com fita métrica inelástica, tendo como ponto de referência o ponto médio entre a costela inferior e a crista ilíaca.

Realizaram-se as medidas de pressão arterial por meio de aparelho automático (Omron model HEM-712C, Omron Health Care, Inc, USA), aceito pela American Heart Association para fins de estudos epidemiológicos. Três medidas de pressão arterial foram tomadas com adequação do manguito ao perímetro braquial; após cinco minutos de repouso na posição sentada, com o braço direito apoiado na altura do átrio esquerdo, o manguito foi automaticamente insuflado. A desinsuflação ocorreu a uma velocidade predeterminada pelo equipamento. Consideraram-se como valores finais de pressão sistólica e diastólica aqueles que representaram a média aritmética das duas últimas medidas (em $\mathrm{mmHg}$ ).

Por ocasião do exame físico, avaliaram-se as práticas atuais de atividade física dos indivíduos, utilizando-se a versão reduzida do Questionário Internacional de Atividade Física (IPAQ), validado em 12 países, incluindo o Brasil $(11,12)$.

Obteve-se amostra (jejum) de sangue venoso para determinação de glicose e perfil lipídico (colesterol total, frações e triglicérides). A glicemia foi dosada pelo método da glico-oxidase e as lipoproteínas, por kits enzimáticos.

Para diagnóstico de síndrome metabólica, utilizou-se o critério da Federação Internacional de Diabetes (13) que considera com síndrome metabólica aqueles com obesidade central (cintura $\geq 90$ ou $\geq 85 \mathrm{~cm}$ para japoneses do sexo masculino e feminino, respectivamente) e de, pelo menos, dois dos seguintes componentes: glicemia de jejum $\geq 100 \mathrm{mg} / \mathrm{dL}$, triglicérides $\geq 150$ $\mathrm{mg} / \mathrm{dL}, \mathrm{HDL}<40 \mathrm{mg} / \mathrm{dL}$ ou $50 \mathrm{mg} / \mathrm{dL}$ (homem e mulher, respectivamente) e pressão arterial sistólica/ diastólica $\geq 130 / 85 \mathrm{mmHg}$ e ou diagnóstico prévio de diabetes, hipertensão e dislipidemia.

Após a obtenção dos dados basais, os participantes foram agendados para as consultas nutricionais (participantes e seus familiares), nas quais receberam orientações sobre as práticas relativas ao estilo de vida saudável.

\section{ESTRATÉGIA DE INTERVENÇÃO NUTRICIONAL}

A intervenção nutricional foi baseada no plano nutricional previamente aplicado em estudo desenvolvido em Unidade Básica de Saúde no Estado de São Paulo (14). A prescrição dietética foi individualizada, considerando as necessidades energéticas e o estado nutricional dos indivíduos. Para os nipo-brasileiros com sobrepeso ou obesidade central, foi prescrita dieta com restrição calórica (redução de 500 a 1.000 das necessidades calorias diárias), de forma a promover perda de peso de $5 \%$. As dietas foram fracionadas em cinco a seis refeições diárias com a seguinte distribuição das calorias totais: $50 \%-60 \%$ provenientes dos carboidratos (com a ingestão dos carboidratos complexos, principalmente); menos de $10 \%$ de ácidos graxos saturados (reduzindo o consumo de carne vermelha para duas porções médias / dia, consumo de 2-3 porções/dia de laticínios pobres em gorduras e desestimulando a ingestão de frituras, banha, maionese, molhos prontos, gordura aparente das carnes, pele de frango e ingestão excessiva de ovos); no mínimo $20 \%$ provenientes de ácidos graxos monoinsaturados e poli-insaturados (com a ingestão de azeite de oliva, oleaginosas e peixes); ingestão de $400 \mathrm{~g} /$ dia de frutas e legumes (equivalendo a 5 porções de frutas, verduras e legumes).

O plano de intervenção foi dividido em duas etapas. A primeira, composta por um atendimento nutricional individualizado, e a segunda, por duas dinâmicas de grupo (primeiro e segundo ano de seguimento) coordenadas por nutricionistas. A consulta nutricional individualizada, com duração média de uma hora, constou de coleta de recordatório alimentar de 24 horas, avaliação nutricional completa (consumo alimentar, necessidades calóricas, estado nutricional, exames clínicos e bioquímicos), prescrição dietética com lista de substituição dos alimentos e orientações sobre a dieta saudável e estímulo à prática de atividade física de, no mínimo, 30 minutos cinco vezes na semana, de acordo com as recomendações da Organização Mundial de Saúde (15). 
Nas duas sessões em grupo, houve dinâmica focada nas metas dietéticas, prática de atividades físicas, adesão ao estilo de vida saudável e discussão das dificuldades relatadas pelos participantes em aderir à intervenção. Utilizou-se como apoio material educativo que constou de: 1) figuras de alimentos de acordo com as funções dos respectivos nutrientes (carboidratos, gorduras, proteínas, vitaminas e minerais); 2) quadros nas cores verde, amarela e vermelha, simbolizando o semáforo; 3 ) modelos de alimentos em formato real para fixação do tamanho das porções; 4) folder ("Dicas para uma vida saudável”) elaborado especificamente para esse fim. Essa etapa contou com a participação de preparadores físicos e nutricionistas.

Nos três momentos de avaliação (basal, em 2005, intermediário, em 2006, e final, em 2007), obtiveram-se três recordatórios de 24 horas, aplicados por equipe de nutricionistas, durante encontros em grupo, exames laboratoriais ou consultas clínicas. Solicitaram-se informações detalhadas dos alimentos e bebidas consumidos nas últimas 24 horas. Todos os questionários foram revisados para controle de qualidade dos dados obtidos. Os alimentos ou preparações foram transformados de medidas caseiras (forma obtida durante a coleta da informação) em gramas ou miligramas. Padronizaram-se todas as receitas e preparações de forma a evitar discrepâncias no momento da digitação dos dados, segundo manual de codificação previamente elaborado. Utilizou-se o programa NutWin ${ }^{\circledR}$ (NutWin Software, Programa de Apoio à Nutrição, Versão 1.5, Escola Paulista de Medicina, São Paulo, Brasil, 2002) para cálculo dos nutrientes ingeridos. Utilizaram-se tabelas de composição de alimentos nacionais e internacionais para os alimentos não contemplados no referido programa (16-18). De forma a avaliar as possíveis mudanças no hábito alimentar, utilizaram-se em cada momento de avaliação os valores médios dos nutrientes citados nos três recordatórios de 24 horas.

\section{ANÁLISE ESTATÍSTICA}

Os dados referentes aos exames laboratoriais, antropométricos e dietéticos foram armazenados em um banco de dados e posteriormente analisados com auxílio do programa SPSS versão 10.0 (SPSS Inc., Chicago, IL, USA). A ingestão de nutrientes foi expressa em gramas e também ajustada para energia total pelo método residual (19).

As variáveis dietéticas e de atividade física foram transformadas antes da análise estatística, usando loga- ritmos naturais, uma vez que não apresentaram distribuição normal. Empregou-se o teste de $t$ de Student para a comparação, por sexo, dos valores médios basais das variáveis bioquímicas, antropométricas e consumo alimentar entre os indivíduos sem e com síndrome metabólica. Utilizou-se o teste $t$ de Student pareado para comparar, por sexo, os valores médios das variáveis de interesse após o segundo ano de intervenção (2005 e 2007) e a presença de síndrome metabólica. Em análise com modelos de regressão linear múltiplos, verificou-se, para cada variável dependente isoladamente, a existência de relações entre as mudanças nos valores de variáveis bioquímicas, antropométricas e consumo alimentar observadas no período de estudo (variáveis dependentes) e a idade, diagnóstico da SM em 2005 e valores basais dessas variáveis. $\mathrm{O}$ valor de $\mathrm{p}<0,05$ foi adotado como significante.

\section{RESULTADOS}

Entre os 360 indivíduos incluídos no presente estudo, observaram-se proporções semelhantes de homens $(45 \%, \mathrm{n}=160)$ e mulheres $(55 \%, \mathrm{n}=200)$. Em análise estratificada por sexo, a idade média dos homens foi estatisticamente maior que a observada entre as mulheres $(56,4$ anos vs. 54,1 anos, respectivamente, $\mathrm{p}=$ 0,042 ). A prevalência da $S M$, em 2005 , foi de $51,5 \%$ (IC 95\%: 44,3\%-58,6\%), entre as mulheres, e 61,9\% (IC 95\%: 53,8\%-69,4\%) nos homens, enquanto, em 2007, esses valores foram de 50,5\% (IC 95\%: 43,3\%-55,6\%) e 53,8\% (IC 95\%: 45,7\%-61,6\%), respectivamente. Em 2007 , identificaram-se 50 casos novos de SM (32\%) entre aqueles que, em 2005, foram classificados como sem SM $(\mathrm{n}=158)$. Em contrapartida, entre os considerados como com SM, em 2005 ( $\mathrm{n}=202), 48$ nipo-brasileiros (24\%) foram considerados como sem SM em 2007.

No momento basal (2005), em ambos os sexos, as médias de HDL-colesterol foram maiores entre aqueles sem síndrome metabólica quando comparados aos com síndrome metabólica, enquanto as do peso corporal, índice de massa corporal, circunferência da cintura, pressão arterial sistólica e diastólica, glicemia de jejum e triglicérides foram maiores entre os indivíduos com síndrome metabólica quando comparados aos sem síndrome. Essas diferenças se mantiveram ao serem comparados os valores dessas variáveis entre os com e sem SM, em 2007 (dados não apresentados). Nenhuma diferença foi observada entre os grupos em relação à prática de atividade física. As mulheres com síndrome 
metabólica eram, em média, mais velhas que as sem síndrome metabólica $(\mathrm{p}=0,006)$ (Tabelas 1 e 2).

Na tabela 1, encontram-se os valores médios das variáveis antropométricas, clínicas e bioquímicas no início do estudo e após dois anos de intervenção, para o sexo masculino, segundo presença da síndrome metabólica. Comparando-se os valores basais e finais ( $1^{\mathrm{a}}$ vs. $3^{\mathrm{a}}$ avaliação), verificaram-se reduções estatisticamente significantes nos grupos com e sem síndrome metabólica em relação ao peso corporal, índice de massa corporal, circunferência da cintura, pressão arterial, colesterol total e frações.
Os valores médios da glicemia de jejum foram estatisticamente menores na avaliação final $\left(1^{\mathrm{a}}\right.$ vs. $3^{\mathrm{a}}$ avaliação $)$ apenas entre aqueles sem síndrome metabólica.

No sexo feminino, verificaram-se reduções estatisticamente significantes ( $1^{\mathrm{a}}$ vs. $3^{\mathrm{a}}$ avaliação) nos valores da circunferência da cintura, pressão arterial e colesterol total, e frações em ambos os grupos (com e sem síndrome metabólica). Mulheres com síndrome metabólica também apresentaram reduções nos valores médios do peso corporal, índice de massa corporal e glicemia de jejum (Tabela 2).

Tabela 1. Valores médios (desvios-padrão) das variáveis antropométricas, clínicas e laboratoriais de nipo-brasileiros do sexo masculino sem e com síndrome metabólica que participaram no programa de intervenção. Bauru, 2007

\begin{tabular}{|c|c|c|c|c|}
\hline Variável & SM & $\begin{array}{c}\text { Basal (1ª avaliação) } \\
\text { Média (DP) }\end{array}$ & $\begin{array}{l}\text { Final (3a. avaliação) } \\
\text { Média (DP) }\end{array}$ & $\begin{array}{c}\text { Valor de } \mathrm{p}^{\dagger} \text { entre } \\
\text { avaliações } \\
\left(1^{\mathrm{a}} \text {. vs. } 3^{\mathrm{a}} \text {.) }\right.\end{array}$ \\
\hline \multirow[t]{2}{*}{ Idade (anos) } & $\operatorname{Sim}$ & $56,1(11,8)$ & & \\
\hline & Não & $56,9(11,3)$ & & \\
\hline Valor de p (com e sem SM em 2005) & 0,638 & & & \\
\hline \multirow[t]{2}{*}{ Peso corporal (kg) } & Sim & $75,3(10,8)$ & $73,5(11,2)$ & $<0,001$ \\
\hline & Não & $62,1(8,3)$ & $60,4(8,6)$ & $<0,001$ \\
\hline Valor de p (com e sem SM em 2005) & $<0,001$ & & & \\
\hline \multirow[t]{2}{*}{ Índice de massa corporal $\left(\mathrm{kg} / \mathrm{m}^{2}\right)$} & Sim & $27,4(3,0)$ & $26,8(3,1)$ & $<0,001$ \\
\hline & Não & $23,1(2,7)$ & $22,5(2,8)$ & $<0,001$ \\
\hline Valor de p (com e sem SM em 2005) & $<0,001$ & & & \\
\hline \multirow[t]{2}{*}{ Circunferência da cintura (cm) } & Sim & $98,0(7,0)$ & $95,5(8,4)$ & $<0,001$ \\
\hline & Não & $86,0(7,4)$ & $83,1(8,4)$ & 0,002 \\
\hline Valor de p (com e sem SM em 2005) & $<0,001$ & & & \\
\hline \multirow[t]{2}{*}{ PAS (mmHg) } & Sim & $146,2(20,3)$ & $136,7(17,4)$ & $<0,001$ \\
\hline & Não & $138,8(20,3)$ & $130,0(18,8)$ & $<0,001$ \\
\hline Valor de p (com e sem SM em 2005) & 0,025 & & & \\
\hline \multirow[t]{2}{*}{ PAD (mmHg) } & $\operatorname{Sim}$ & $86,6(12,2)$ & $79,7(11,9)$ & $<0,001$ \\
\hline & Não & $80,6(10,5)$ & $75,4(10,7)$ & $<0,001$ \\
\hline Valor de p (com e sem SM em 2005) & $<0,001$ & & & \\
\hline \multirow[t]{2}{*}{ Glicemia de jejum (mg/dl) } & $\operatorname{Sim}$ & $125,6(35,1)$ & $119,8(35,7)$ & 0,061 \\
\hline & Não & $109,4(19,8)$ & $104,5(18,5)$ & 0,018 \\
\hline Valor de p (com e sem SM em 2005) & $<0,001$ & & & \\
\hline \multirow[t]{2}{*}{ Colesterol total (mg/dl) } & Sim & $215,8(45,5)$ & $186,3(39,4)$ & $<0,001$ \\
\hline & Não & $202,1(48,1)$ & $182,2(39,0)$ & $<0,001$ \\
\hline Valor de p (com e sem SM em 2005) & 0,066 & & & \\
\hline \multirow[t]{2}{*}{ LDL-colesterol (mg/dl) } & Sim & $125,4(37,3)$ & $102,0(31,2)$ & $<0,001$ \\
\hline & Não & $124,1(41,1)$ & $109,5(32,0)$ & $<0,001$ \\
\hline Valor de p (com e sem SM em 2005) & 0,683 & & & \\
\hline \multirow[t]{2}{*}{ HDL-colesterol (mg/dl) } & Sim & $47,7(10,8)$ & $44,4(10,8)$ & 0,001 \\
\hline & Não & $53,9(13,7)$ & $50,1(12,0)$ & 0,008 \\
\hline Valor de p (com e sem SM em 2005) & 0,002 & & & \\
\hline \multirow[t]{2}{*}{ Triglicérides (mg/dl) } & Sim & $214,7(153,5)$ & $191,2(106,1)$ & 0,135 \\
\hline & Não & $137,6(102,3)$ & $134,5(88,1)$ & 0,816 \\
\hline Valor de p (com e sem SM em 2005) & $<0,001$ & & & \\
\hline
\end{tabular}

SM: síndrome metabólica; IMC: índice de massa corporal; PAS: pressão arterial sistólica; PAD: pressão arterial diastólica; † Valor de p referente ao teste $t$ de Student pareado. 
Tabela 2. Valores médios (desvios-padrão) das variáveis antropométricas, clínicas e laboratoriais de nipo-brasileiros do sexo feminino sem e com síndrome metabólica que participaram no programa de intervenção. Bauru, 2007

\begin{tabular}{|c|c|c|c|c|}
\hline Variável & SM & $\begin{array}{c}\text { Basal (1ª avaliação) } \\
\text { Média (DP) }\end{array}$ & $\begin{array}{l}\text { Final (3a. avaliação) } \\
\text { Média (DP) }\end{array}$ & $\begin{array}{c}\text { Valor de } \mathrm{p}^{\dagger} \text { entre } \\
\text { avaliações } \\
\left(1^{\mathrm{a}} \text {. vs. } 3^{\mathrm{a}} .\right)\end{array}$ \\
\hline \multirow[t]{2}{*}{ Idade (anos) } & $\operatorname{Sim}$ & $56,3(10,0)$ & & \\
\hline & Não & $52,1(11,1)$ & & \\
\hline Valor de $p$ (com e sem SM em 2005) & 0,006 & & & \\
\hline \multirow[t]{2}{*}{ Peso corporal (kg) } & Sim & $61,0(8,0)$ & $60,0(8,3)$ & 0,005 \\
\hline & Não & $50,8(6,5)$ & $50,9(7,4)$ & 0,666 \\
\hline Valor de $p$ (com e sem SM em 2005) & $<0,001$ & & & \\
\hline \multirow[t]{2}{*}{ Índice de massa corporal $\left(\mathrm{kg} / \mathrm{m}^{2}\right)$} & $\operatorname{Sim}$ & $26,6(3,1)$ & $26,1(3,3)$ & 0,005 \\
\hline & Não & $21,8(2,7)$ & $21,8(3,0)$ & 0,640 \\
\hline Valor de p (com e sem SM em 2005) & $<0,001$ & & & \\
\hline \multirow[t]{2}{*}{ Circunferência da cintura (cm) } & Sim & $93,0(7,4)$ & $88,4(8,7)$ & $<0,001$ \\
\hline & Não & $80,9(7,5)$ & $78,6(8,7)$ & $<0,001$ \\
\hline Valor de $p$ (com e sem SM em 2005) & $<0,001$ & & & \\
\hline \multirow[t]{2}{*}{ PAS (mmHg) } & $\operatorname{Sim}$ & $144,1(23,4)$ & $135,7(17,5)$ & $<0,001$ \\
\hline & Não & $127,3(19,6)$ & $122,0(17,5)$ & $<0,001$ \\
\hline Valor de p (com e sem SM em 2005) & $<0,001$ & & & \\
\hline \multirow[t]{2}{*}{ PAD (mmHg) } & $\operatorname{Sim}$ & $83,4(11,2)$ & $77,9(10,2)$ & $<0,001$ \\
\hline & Não & $75,8(11,6)$ & $72,6(10,0)$ & $<0,001$ \\
\hline Valor de $p$ (com e sem SM em 2005) & $<0,001$ & & & \\
\hline \multirow[t]{2}{*}{ Glicemia de jejum (mg/dl) } & Sim & $116,4(34,7)$ & $111,3(27,7)$ & 0,037 \\
\hline & Não & $95,2(11,3)$ & $96,1(14,2)$ & 0,426 \\
\hline Valor de $p$ (com e sem SM em 2005) & $<0,001$ & & & \\
\hline \multirow[t]{2}{*}{ Colesterol Total (mg/dl) } & $\operatorname{Sim}$ & $226,4(40,6)$ & $193,7(35,1)$ & $<0,001$ \\
\hline & Não & $217,3(61,4)$ & $189,4(35,1)$ & $<0,001$ \\
\hline Valor de $p$ (com e sem SM em 2005) & 0,227 & & & \\
\hline \multirow[t]{2}{*}{ LDL-colesterol (mg/dl) } & Sim & $134,8(37,7)$ & $108,6(31,5)$ & $<0,001$ \\
\hline & Não & $131,8(36,7)$ & $110,2(31,2)$ & $<0,001$ \\
\hline Valor de p (com e sem SM em 2005) & 0,494 & & & \\
\hline \multirow[t]{2}{*}{ HDL-colesterol (mg/dl) } & Sim & $52,9(10,6)$ & $48,3(11,7)$ & $<0,001$ \\
\hline & Não & $61,0(11,1)$ & $57,0(12,1)$ & $<0,001$ \\
\hline Valor de $p$ (com e sem SM em 2005) & $<0,001$ & & & \\
\hline \multirow[t]{2}{*}{ Triglicérides (mg/dl) } & $\operatorname{Sim}$ & $187,2(115,0)$ & $195,4(167,7)$ & 0,661 \\
\hline & Não & $111,1(74,2)$ & $110,2(52,9)$ & 0,876 \\
\hline Valor de p (com e sem SM em 2005) & $<0,001$ & & & \\
\hline
\end{tabular}

SM: síndrome metabólica; IMC: índice de massa corporal; PAS: pressão arterial sistólica; PAD: pressão arterial diastólica; † Valor de p referente ao teste $t$ de Student pareado.

Apesar de ter-se estimulado a prática de atividade física durante o programa de intervenção, não foram observadas diferenças estatisticamente significantes em relação a essa variável quando se compararam os valores basais e finais em ambos os grupos e sexo (dados não apresentados).

Em relação às mudanças do consumo alimentar, as comparações dos valores basais com os finais $\left(1^{\mathrm{a}} v s .3^{\mathrm{a}}\right.$ avaliação) mostraram que os homens sem síndrome metabólica diminuíram a ingestão de gorduras totais, ácidos graxos saturados, ácidos graxos monoinsaturados e colesterol e aumentaram a ingestão de fibra total (Tabela 3). Considerando apenas os dados de 2007, os homens sem SM apresentaram valores médios estatisticamente maiores da ingestão de fibra total quando comparados aos com essa síndrome $(30,1(14,3)$ vs. 26, $7(11,2) \mathrm{g} /$ dia, $\mathrm{p}<0,05)$.

Para as mulheres, conforme se verifica na tabela 4 , no momento basal (2005), diferenças estatisticamente 
significantes foram observadas nos valores médios das variáveis dietéticas (calorias totais e proteínas), sendo estes menores entre as com síndrome metabólica quando comparadas às sem essa condição. Além disso, verificaram-se reduções estatisticamente significantes no grupo sem síndrome metabólica em relação à ingestão de gorduras totais e ácidos graxos monoinsaturados.

Em análise com o modelo de regressão linear múltipla, verificaram-se as diferenças encontradas em análise bruta; para o peso corporal, índice de massa corporal e circunferência da cintura (entre o sexo masculino), foram independentes da idade, valores dessas variáveis em 2005 e a presença de SM no início do estudo, enquanto a redução da pressão arterial sistólica foi maior entre aqueles com SM e que apresentavam menores valores de PAS em 2005. Por outro lado, a redução da pressão arterial diastólica e colesterol total foi maior entre os mais jovens e que, em 2005, apresentavam menores valores dessas variáveis. Em relação à redução da glicemia de jejum foi independente da idade e presença de SM, mas foi maior entre os que apresentavam menores valores em 2005. Para o LDL-colesterol e HDL-colesterol, as diferenças foram maiores entre os sem SM e que, em 2005, apresentavam menores valores dessas variáveis (Tabela 5 ).

Tabela 3. Valores médios (desvios-padrão) das variáveis dietéticas de nipo-brasileiros do sexo masculino sem e com síndrome metabólica que participaram no programa de intervenção. Bauru, 2007

\begin{tabular}{|c|c|c|c|c|}
\hline Variável & SM & $\begin{array}{l}\text { Basal (1ª avaliação) } \\
\text { Média (DP) }\end{array}$ & $\begin{array}{l}\text { Final (3a. avaliação) } \\
\text { Média (DP) }\end{array}$ & $\begin{array}{l}\text { Valor de } \mathrm{p}^{\dagger} \text { entre } \\
\text { avaliaçõ̃es } \\
\left(1^{\mathrm{a}} \text {. vs. } 3^{\mathrm{a}} \text {.) }\right.\end{array}$ \\
\hline \multirow[t]{2}{*}{ Calorias totais } & $\operatorname{Sim}$ & $2376(897,4)$ & $2305(859,8)$ & 0,491 \\
\hline & Não & $2223(721,7)$ & $2201(751,8)$ & 0,554 \\
\hline Valor de p (com e sem SM em 2005) & 0,331 & & & \\
\hline \multirow[t]{2}{*}{ Carboidratos (g) } & $\operatorname{Sim}$ & $296,5(109,2)$ & $290,1(100,9)$ & 0,870 \\
\hline & Não & $295,5(106,5)$ & $298,5(105,8)$ & 0,656 \\
\hline Valor de p (com e sem SM em 2005) & 0,126 & & & \\
\hline \multirow[t]{2}{*}{ Proteína (g) } & Sim & $105,9(49,0)$ & $100,1(41,2)$ & 0,689 \\
\hline & Não & $95,7(36,1)$ & $92,8(38,2)$ & 0,075 \\
\hline Valor de p (com e sem SM em 2005) & 0,780 & & & \\
\hline \multirow[t]{2}{*}{ Gordura total (g) } & $\operatorname{Sim}$ & $83,4(47,1)$ & $78,8(47,8)$ & 0,200 \\
\hline & Não & $71,3(32,5)$ & $68,0(33,0)$ & 0,054 \\
\hline Valor de p (com e sem SM em 2005) & 0,165 & & & \\
\hline \multirow[t]{2}{*}{ Ác. g. monoinsaturado (g) } & $\operatorname{Sim}$ & $29,7(18,9)$ & $27,4(19,5)$ & 0,158 \\
\hline & Não & $24,9(12,8)$ & $22,3(10,7)$ & 0,009 \\
\hline Valor de p (com e sem SM em 2005) & 0,212 & & & \\
\hline \multirow[t]{2}{*}{ Ac. g. poli-insaturado (g) } & $\operatorname{Sim}$ & $20,3(10,3)$ & $20,5(11,0)$ & 0,823 \\
\hline & Não & $17,7(8,1)$ & $19,2(10,8)$ & 0,247 \\
\hline Valor de p (com e sem SM em 2005) & 0,289 & & & \\
\hline \multirow[t]{2}{*}{ Ac. g. saturado $(g)$} & $\operatorname{Sim}$ & $24,5(16,4)$ & $22,7(15,8)$ & 0,197 \\
\hline & Não & $21,0(11,6)$ & $18,9(10,2)$ & 0,036 \\
\hline Valor de p (com e sem SM em 2005) & 0,368 & & & \\
\hline \multirow[t]{2}{*}{ Colesterol (mg) } & Sim & $283,7(188,7)$ & $252,3(157,9)$ & 0,238 \\
\hline & Não & $246,8(135,1)$ & $210,0(110,2)$ & 0,040 \\
\hline Valor de p (com e sem SM em 2005) & 0,635 & & & \\
\hline \multirow[t]{2}{*}{ Fibra total (g) } & $\operatorname{Sim}$ & $26,3(12,3)$ & $27,4(11,6)$ & 0,362 \\
\hline & Não & $25,8(12,7)$ & $29,3(14,0)$ & 0,013 \\
\hline Valor de p (com e sem SM em 2005) & 0,829 & & & \\
\hline \multirow[t]{2}{*}{ Álcool (g) } & $\operatorname{Sim}$ & $4,0(10,1)$ & $5,4(11,6)$ & 0,500 \\
\hline & Não & $6,3(12,8)$ & $5,0(9,9)$ & 0,524 \\
\hline Valor de p (com e sem SM em 2005) & 0,786 & & & \\
\hline
\end{tabular}

SM: síndrome metabólica; dados de macronutrientes ajustados por energia total e sexo. ${ }^{\dagger}$ Valor de p referente ao teste $t$ de Student pareado. 
Tabela 4. Valores médios (desvios-padrão) das variáveis dietéticas de nipo-brasileiros do sexo feminino sem e com síndrome metabólica que participaram no programa de intervenção. Bauru, 2007

\begin{tabular}{|c|c|c|c|c|}
\hline Variável & SM & $\begin{array}{l}\text { Basal (1 }{ }^{\text {a }} \text {. avaliação) } \\
\text { Média (DP) }\end{array}$ & $\begin{array}{l}\text { Final (3a. avaliação) } \\
\text { Média (DP) }\end{array}$ & $\begin{array}{c}\text { Valor de } \mathrm{p}^{\dagger} \text { entre } \\
\text { avaliações } \\
\left(1^{\mathrm{a}} \text {. vs. } 3^{\mathrm{a}} .\right)\end{array}$ \\
\hline \multirow[t]{2}{*}{ Calorias totais } & $\operatorname{Sim}$ & $1549(482,4)$ & $1523(432,1)$ & 0,360 \\
\hline & Não & $1735(545,4)$ & $1680(687,4)$ & 0,424 \\
\hline Valor de p (com e sem SM em 2005) & 0,013 & & & \\
\hline \multirow[t]{2}{*}{ Carboidratos (g) } & Sim & $209,0(68,1)$ & $206,7(66,5)$ & 0,817 \\
\hline & Não & $233,4(74,2)$ & $227,5(91,9)$ & 0,726 \\
\hline Valor de p (com e sem SM em 2005) & 0,523 & & & \\
\hline \multirow{2}{*}{ Proteína (g) } & Sim & $67,1(22,4)$ & $66,3(22,2)$ & 0,439 \\
\hline & Não & $71,6(27,6)$ & $68,2(29,1)$ & 0,508 \\
\hline Valor de p (com e sem SM em 2005) & 0,053 & & & \\
\hline \multirow[t]{2}{*}{ Gordura total (g) } & Sim & $51,9(23,1)$ & $49,2(20,8)$ & 0,124 \\
\hline & Não & $59,6(23,0)$ & $52,9(23,7)$ & 0,003 \\
\hline Valor de p (com e sem SM em 2005) & 0,270 & & & \\
\hline \multirow[t]{2}{*}{ Ác. g. monoinsaturado (g) } & $\operatorname{Sim}$ & $17,5(8,6)$ & $16,6(7,4)$ & 0,650 \\
\hline & Não & $20,6(8,6)$ & $17,5(8,3)$ & 0,002 \\
\hline Valor de p (com e sem SM em 2005) & 0,119 & & & \\
\hline \multirow[t]{2}{*}{ Ac. g. poli-insaturado (g) } & $\operatorname{Sim}$ & $13,4(6,2)$ & $13,0(5,7)$ & 0,439 \\
\hline & Não & $15,6(6,5)$ & $14,2(7,0)$ & 0,066 \\
\hline Valor de p (com e sem SM em 2005) & 0,217 & & & \\
\hline \multirow[t]{2}{*}{ Ac. g. saturado (g) } & $\operatorname{Sim}$ & $15,1(8,4)$ & $14,5(7,7)$ & 0,811 \\
\hline & Não & $17,5(7,7)$ & $15,7(7,7)$ & 0,077 \\
\hline Valor de p (com e sem SM em 2005) & 0,230 & & & \\
\hline \multirow[t]{2}{*}{ Colesterol (mg) } & Sim & $176,3(100,3)$ & $167,1(106,9)$ & 0,485 \\
\hline & Não & $206,3(117,2)$ & $179,0(89,0)$ & 0,685 \\
\hline Valor de p (com e sem SM em 2005) & 0,857 & & & \\
\hline \multirow[t]{2}{*}{ Fibra total (g) } & Sim & $18,3(7,5)$ & $18,6(8,9)$ & 0,241 \\
\hline & Não & $19,4(9,2)$ & $20,3(9,9)$ & 0,571 \\
\hline Valor de p (com e sem SM em 2005) & 0,324 & & & \\
\hline \multirow[t]{2}{*}{ Álcool (g) } & Sim & $0,1(0,8)$ & $0,3(1,3)$ & 0,116 \\
\hline & Não & $0,4(1,9)$ & $0,6(3,8)$ & 0,248 \\
\hline Valor de p (com e sem SM em 2005) & 0,818 & & & \\
\hline
\end{tabular}

SM: síndrome metabólica; dados de macronutrientes ajustados por energia total e sexo. ${ }^{\dagger}$ Valor de $p$ referente ao teste $t$ de Student pareado.

Para o sexo feminino, verificou-se que as diferenças encontradas para o peso corporal e índice de massa corporal foram independentes da idade, valor dessas variáveis em 2005 e a presença de SM no início do estudo. A redução da circunferência da cintura foi maior entre aqueles que, em 2005, apresentavam menores valores de cintura, enquanto as reduções da pressão arterial sistólica e da glicemia de jejum foram maiores entre as mais velhas. Por outro lado, a redução da pressão arterial diastólica foi independente da idade e maior entre aqueles que, em 2005, apresentavam menores valores de PAD. As reduções do colesterol total, LDL-colesterol e HDL-colesterol foram independentes da idade e presença de SM no início do estudo e maior entre aqueles que, em 2005, apresentavam menores valores dessas variáveis (Tabela 5 ).

As diferenças encontradas na redução da ingestão da gordura total, em ambos os sexos, foram maiores entre os mais jovens, com SM e com menor ingestão desse nutriente em 2005. Por outro lado, a redução do ácido graxo monoinsaturado foi independente da presença de SM (Tabela 6). 
Tabela 5. Coeficiente de regressão $(\beta)$ das mudanças das variáveis bioquímicas e antropométricas de nipo-brasileiros por sexo que participaram no programa de intervenção. Bauru, 2007

\begin{tabular}{|c|c|c|c|c|}
\hline \multirow[b]{2}{*}{ Sexo } & \multirow[b]{2}{*}{ Variável dependente } & \multicolumn{3}{|c|}{ Valor de $\beta$ (IC 95\%) } \\
\hline & & Idade & Diagnóstico SM em 2005 & $\begin{array}{c}\text { Valor da variável } \\
\text { Dependente em } 2005\end{array}$ \\
\hline \multirow[t]{10}{*}{ Masculino } & Diferença do peso corporal & $0,061(-0,28-0,055)$ & $-0,080(-1,396-0,538)$ & $-0,012(-0,047-0,042)$ \\
\hline & Diferença do índice de massa corporal & $0,033(-0,012-0,018)$ & $-0,075(-0,522-0,221)$ & $-0,042(-0,064-0,041)$ \\
\hline & Diferença da circunferência da cintura & $-0,129(-0,131-0,016)$ & $-0,065(-1,227-2,619)$ & $-0,131(-0,171-0,029)$ \\
\hline & Diferença da PAS & $0,104(-0,067-0,368)$ & $0,157(0,413-10,579)$ & $-0,539(-0,619--0,347)$ \\
\hline & Diferença da PAD & $-0,176(-0,257-0,021)$ & $0,103(-0,879-4,818)$ & $-0,492(-0,511--0,268)$ \\
\hline & Diferença da glicemia de jejum & $0,035(-0,221-0,362)$ & $0,097(-2,728-11,994)$ & $-0,432(-0,459--0,218)$ \\
\hline & Diferença do colesterol total & $-0,135(-0,854--0,028)$ & $-0,063(-14,609-4,837)$ & $-0,613(-0,591--0,389)$ \\
\hline & Diferença do LDL-colesterol & $-0,080(-0,607-0,143)$ & $-0,170(-20,705--2,849)$ & $-0,651(-0,693--0,463)$ \\
\hline & Diferença do HDL-colesterol & $-0,101(-0,213-0,032)$ & $-0,165(-6,619-0,424)$ & $-0,540(-0,564--0,324)$ \\
\hline & Diferença do triglicérides & $-0,045(-1,74-0,732)$ & $0,191(20,143-82,109)$ & $-0,789(-0,868-0,642)$ \\
\hline \multirow[t]{10}{*}{ Feminino } & Diferença do peso corporal & $-0,040(-0,48-0,028)$ & $-0,128(-1,562-0,190)$ & $0,038(-0,038-0,061)$ \\
\hline & Diferença do índice de massa corporal & $-0,054(-0,022-0,010)$ & $-0,109(-0,640-0,130)$ & $0,006(-0,049-0,052)$ \\
\hline & Diferença da circunferência da cintura & $-0,102(-0,131-0,024)$ & $0,000(-1,767-1,769)$ & $-0,192(-0,207--0,020)$ \\
\hline & Diferença da PAS & $0,199(0,122-0,511)$ & $0,092(-1,298-7,637)$ & $-0,714(-0,635--0,433)$ \\
\hline & Diferença da PAD & $0,004(-0,105-0,111)$ & $-0,028(-3,012-1,966)$ & $-0,533(-0,512--0,310)$ \\
\hline & Diferença da glicemia de jejum & $0,170(0,088-0,486)$ & $0,010(-4,303-5,038)$ & $-0,566(-0,460--0,293)$ \\
\hline & Diferença do colesterol total & $0,050(-0,199-0,670)$ & $-0,030(-12,332-6,305)$ & $-0,774(-0,826-0,652)$ \\
\hline & Diferença do LDL-colesterol & $0,025(-0,297-0,454)$ & $-0,078(-13,712-2,745)$ & $-0,638(-0,718--0,499)$ \\
\hline & Diferença do HDL-colesterol & $0,050(-0,083-0,177)$ & $-0,139(-6,000-0,310)$ & $-0,391(-0,479--0,213)$ \\
\hline & Diferença do triglicérides & $0,005(-1,489-1,602)$ & $0,184(11,162-81,659)$ & $-0,442(-0,710-0,374)$ \\
\hline
\end{tabular}

SM: síndrome metabólica; PAS: pressão arterial sistólica; PAD: pressão arterial diastólica.

Em relação às reduções da ingestão do colesterol total e do ácido graxo saturado (sexo masculino), essas foram maiores entre aqueles que, em 2005, eram mais jovens e apresentavam menor consumo desses nutrientes. O aumento da ingestão da fibra total foi maior entre aqueles que, em 2005, eram mais jovens e apresentavam menor ingestão de fibra (Tabela 6).

\section{DISCUSSÃO}

O presente trabalho avaliou as mudanças nos perfis metabólico e nutricional de nipo-brasileiros com e sem síndrome metabólica, após dois anos de participação em programa de intervenção sobre o estilo de vida. Também foi uma oportunidade de contribuir com a literatura atualmente escassa de programa de intervenção comunitário e síndrome metabólica em países em desenvolvimento.

Ao se considerar a totalidade de indivíduos que completaram as três avaliações do estudo de intervenção, verificou-se melhora no perfil metabólico e antro- pométrico dos indivíduos nipo-brasileiros com e sem síndrome metabólica, em ambos os gêneros.

De acordo com os resultados observados, as mudanças dietéticas entre os homens sem síndrome metabólica se deram principalmente em relação à melhoria da qualidade das gorduras ingeridas, levando à queda nos valores de gordura total, dos ácidos graxos saturados e do colesterol. Isso reflete o impacto positivo das metas do programa nutricional, principalmente em relação ao incentivo na redução da ingestão de carnes ricas em gordura saturada, laticínios integrais, frituras, banha e maionese. Além disso, o incentivo ao consumo de legumes, frutas e cereais integrais pode ter beneficiado no ligeiro aumento da ingestão de fibra total entre os homens sem síndrome metabólica. Essas mudanças podem sugerir que esse grupo seja mais consciente de seu comportamento alimentar e busque maiores informações sobre saúde e alimentação. O estudo aponta que a percepção e o conhecimento refletem nas escolhas e atitudes alimentares mais salutares. Uma possível explicação da ligeira mudança observada entre as mu- 
Tabela 6. Coeficiente de regressão $(\beta)$ das mudanças das variáveis de consumo de nipo-brasileiros por sexo que participaram no programa de intervenção. Bauru, 2007

\begin{tabular}{|c|c|c|c|c|}
\hline \multirow[b]{2}{*}{ Sexo } & \multirow[b]{2}{*}{ Variável dependente } & \multicolumn{3}{|c|}{ Valor de $\beta$ (IC 95\%) } \\
\hline & & Idade & Diagnóstico SM em 2005 & $\begin{array}{c}\text { Valor da variável } \\
\text { Dependente em } 2005\end{array}$ \\
\hline \multirow[t]{10}{*}{ Masculino } & Diferença das calorias totais & $-0,291(-29,403-10,278)$ & $0,074(-92,729-336,745)$ & $-0,608(-0,728-0,456)$ \\
\hline & Diferença dos carboidratos & $-0,304(-3,702--1,474)$ & $-0,015(-29,740-23,593)$ & $-0,550(-0,629--0,387)$ \\
\hline & Diferença das proteínas & $-0,219(-1,426--0,360)$ & $-0,063(-5,672-18,101)$ & $-0,707(-0,925--0,636)$ \\
\hline & Diferença da gordura total & $-0,239(-1,454--0,372)$ & $0,131(0,164-23,963)$ & $-0,649(-0,861--0,552)$ \\
\hline & Diferença dos ac. g. monoinsaturados & $-0,226(-0,575--0,129)$ & $0,103(-1,050-8,761)$ & $-0,639(-0,347--0,220)$ \\
\hline & Diferença dos ac. g. poli-insaturados & $-0,196(-0,340--0,053)$ & $0,102(-0,788-5,711)$ & $-0,573(-0,849--0,509)$ \\
\hline & Diferença dos ac. g. saturados & $-0,230(-0,493--0,133)$ & $0,092(-0,982-6,990)$ & $-0,705(-0,927--0,633)$ \\
\hline & Diferença do colesterol & $-0,202(-4,829-1,128)$ & $0,108(-2,346-79,432)$ & $-0,755(-0,928--0,664)$ \\
\hline & Diferença da fibra total & $-0,179(-0,370--0,055)$ & $-0,040(-4,910-2,642)$ & $-0,549(-0,741--0,454)$ \\
\hline & Diferença do álcool & $0,000(-0,108-0,108)$ & $0,150(0,197-5,320)$ & $-0,471(-0,471--0,254)$ \\
\hline \multirow[t]{10}{*}{ Feminino } & Diferença das calorias totais & $-0,079(-12,065-3,064)$ & $-0,010(-169,736-145,007)$ & $-0,495(-0,734-0,427)$ \\
\hline & Diferença dos carboidratos & $-0,021(-1,209-0,872)$ & $0,022(-18,477-25,951)$ & $-0,485(-0,732--0,429)$ \\
\hline & Diferença das proteínas & $-0,045(-0,447-0,213)$ & $0,025(-5,538-8,372)$ & $-0,526(-0,717--0,442)$ \\
\hline & Diferença da gordura total & $-0,207(0,758--0,187)$ & $0,009(-5,333-6,260)$ & $-0,635(-0,803--0,544)$ \\
\hline & Diferença dos ac. g. monoinsaturados & $-0,183(-0,264--0,048)$ & $-0,010(-2,009-2,366)$ & $-0,619(-0,293--0,195)$ \\
\hline & Diferença dos ac. g. poli-insaturados & $-0,105(-0,165-0,009)$ & $0,015(-1,593-2,071)$ & $-0,636(-0,922--0,641)$ \\
\hline & Diferença dos ac. g. saturados & $-0,209(-0,273-0,069)$ & $-0,015(-1,772-2,312)$ & $-0,659(-0,849-0,583)$ \\
\hline & Diferença do colesterol & $0,014(-1,180-1,500)$ & $-0,020(-33,070-23,079)$ & $-0,657(-0,880--0,621)$ \\
\hline & Diferença da fibra total & $0,009(-1,115-0,133)$ & $0,000(-2,674-2,674)$ & $-0,546(-0,862--0,552)$ \\
\hline & Diferença do álcool & $0,004(-0,030-0,032)$ & $-0,063(-0,976-0,369)$ & $-0,313(-0,689--0,271)$ \\
\hline
\end{tabular}

SM: síndrome metabólica.

lheres sem síndrome metabólica - e que pode ter ocorrido antes do programa de intervenção - está associada ao maior conhecimento sobre alimentação saudável e sua relação com a saúde entre o sexo feminino $(20)$.

Por outro lado, não se observaram diferenças dietéticas entre os homens e mulheres com síndrome metabólica. Esses resultados podem indicar que os indivíduos estão sub-relatando a ingestão alimentar ou já estavam num processo prévio de mudança dos hábitos alimentares. O sub-relato da ingestão de energia pode ser um problema quando são investigados indivíduos obesos (21). Há amplas evidências na literatura para se recomendar a redução das calorias totais fornecidas pelas gorduras para o controle da obesidade $(22,23)$. No entanto, é importante salientar que a diminuição da ingestão de gordura $(<30 \%$ das calorias totais) frequentemente implica o aumento da ingestão de carboidrato que, se for excessivo, também contribui para a ocorrência de obesidade (24), fato este não observado nos grupos estudados. Sabe-se que a alta ingestão de gordura (> 35\% das calorias totais) traz modificações no metabolismo que favorecem o acúmulo de gordura corporal (25).

Em estudo prévio realizado com a comunidade nipo-brasileira de Bauru, observou-se, após ajuste para variáveis de controle, que o alto consumo de gordura total (último quinto) se associou ao aumento de cinco vezes na chance de desenvolver síndrome metabólica, quando comparado com o mais baixo consumo (primeiro quinto). Quando os indivíduos foram avaliados por meio dos grupos de alimentos consumidos, observou-se associação da ingestão de frituras à síndrome metabólica, com acréscimo significante do risco ao longo dos quintos (26).

Neste estudo, constatou-se diminuição da ingestão de ácidos graxos saturados entre os homens sem síndrome e ligeira queda nos com síndrome metabólica. Valer reforçar que a estratégia de intervenção nutricional contemplou a melhoria na qualidade da gordura, estimulando os indivíduos a reduzirem a ingestão de alimentos ricos em ácidos graxos saturados e colesterol. 
A seleção adequada do tipo de gordura a ser consumida é importante para minimizar seu efeito deletério nos distúrbios metabólicos que compõem a síndrome metabólica. Reforçando nossos achados, o estudo de intervenção constatou diminuição do índice de massa corporal e adiposidade corporal quando reduzida a ingestão da gordura total, particularmente os ácidos graxos saturados em nipo-americanos pré-diabéticos (27).

Em outro estudo, realizado com a coorte dos nipo-brasileiros, observou-se que entre os homens o consumo de carne vermelha (principal fonte de gordura saturada) aumentou em 4,78 vezes o risco de desenvolver a síndrome metabólica, ao longo dos sete anos de acompanhamento (28).

Houve redução no consumo de colesterol dietético que pode ter sido a causa da redução nos níveis séricos de colesterol total e LDL-colesterol no grupo dos homens sem síndrome metabólica, embora reduções semelhantes não tenham sido observadas nas mulheres. Weggemans e cols. (2001) (29), em estudo realizado a partir das publicações que focalizaram avaliar o efeito do colesterol dietético, verificaram que a redução no consumo de alimentos ricos em colesterol promoveu diminuição na concentração do LDL-colesterol sanguíneo.

Apesar de o presente estudo não diferenciar a qualidade da fibra ingerida, observou-se ligeiro aumento do consumo em homens sem síndrome, o que também pode ter participado positivamente na melhora do perfil lipídico. Estudos recentes indicam que fibras solúveis podem reduzir as concentrações de LDL-colesterol, minimizando a absorção de gordura no trato gastrointestinal. Os resultados de estudo do tipo metanálise mostraram que as fibras solúveis provocaram pequena, mas significativa, redução nas concentrações séricas do colesterol total e do LDL-colesterol (30).

A redução da ingestão dos ácidos graxos saturados e colesterol foram as metas atingidas pelos indivíduos. Por outro lado, não se observou aumento da ingestão de ácidos graxos monoinsaturados, cuja principal fonte, e estimulado seu consumo, foi o azeite de oliva. Essa baixa ingestão pode ter contribuído para a diminuição na concentração sérica do HDL-colesterol em ambos os grupos e gêneros. Estudo de Kasim-Karakas e cols. (31) aponta que, na presença de hipertrigliceridemia ou quando o HDL-colesterol for inferior ao desejável, deve-se aumentar a quantidade de gordura monoinsaturada, reduzindo a oferta de carboidratos. Assim como os ácidos graxos monoinsaturados, os poli-insaturados podem ser benéficos na síndrome metabólica (32).
A ausência de um grupo de controle é a principal limitação observada no presente estudo. Estudos de intervenção que utilizam procedimentos aleatórios são mais adequados para avaliação do efeito causal (33); no entanto, considerando questões de ordem ética e logística, tal procedimento não foi adotado entre esses sujeitos. Como previamente descrito (9), altas prevalências de intolerância à glicose, dislipidemia, hipertensão e obesidade central foram observadas entre esses nipo-brasileiros, e iniciativas urgentes de intervenção eram necessárias para minimizar o problema. Nesse contexto, o JBDSG optou pelo estudo do tipo experimental não controlado.

Além disso, outras limitações devem ser consideradas: 1) sabe-se que os erros de medida não podem ser descartados nas avaliações de consumo alimentar e atividade física; de qualquer forma, a escolha de metodologia padronizada para coleta dessas informações provavelmente minimizou a ocorrência desse vício; 2) sabe-se que a motivação para mudanças de hábitos alimentares e de saúde dos indivíduos que participaram do estudo de intervenção pode ser diferente entre os grupos. Os indivíduos classificados com síndrome metabólica podem ter incorporado mudanças nos hábitos alimentares e de saúde, decorrentes dos diagnósticos prévios das doenças que compõem a síndrome metabólica; 3 ) não foi avaliada a adesão dos grupos incluídos no programa de intervenção; contudo, pode-se supor que, se a adesão ao programa fosse de $100 \%$, os resultados positivos seriam ainda melhores; 4) os resultados encontrados referem-se aos nipo-brasileiros residentes em Bauru e não se conhece o impacto dessa intervenção em outras populações; 5 ) não se sabe se os resultados finais permaneceriam os mesmos, caso os indivíduos excluídos que não completaram as três avaliações fossem incluídos nas análises, pois pode-se supor que a motivação para o cuidado com a saúde nesse grupo seja menor que no grupo analisado.

Os resultados encontrados mostraram impacto positivo do programa de intervenção nos perfis metabólico e nutricional dos nipo-brasileiros com e sem síndrome metabólica. É necessário o desenvolvimento de outros estudos para avaliação da manutenção desses benefícios ao longo do tempo e em outras populações.

Agradecimentos: Esta pesquisa foi apoiada pela Fundação de Amparo à Pesquisa do Estado de São Paulo/Fapesp (05/59178-7). Renata Damião foi bolsista de pós-doutorado da Fapesp (5/60290-6). À participação da comunidade nipo-brasileira de Bauru, que viabilizou a realização deste estudo.

Declaração: os autores declaram não haver conflitos de interesse científico neste estudo. 
Membros do Japanese-Brazilian Diabetes Study Group

Hirai A, Hirai AT, Harima H, Kikuchi M, Osiro K, Gimeno SGA (Departamento de Medicina Preventiva, Universidade Federal de São Paulo, Brasil); Damião R (Departamento de Nutrição, Universidade Federal do Triângulo Mineiro, Brasil); Franco LJ, Sartorelli D (Departamento de Medicina Social, Faculdade de Medicina de Ribeirão Preto, Universidade de São Paulo, Brasil); Matsumura L (Departamento de Medicina Médica, Universidade Federal de São Paulo, Brasil); Wakisaka K (Centro de Estudo Nipo-Brasileiro, Brasil); Chaim R (Curso de Graduação em Nutrição, Universidade Sagrado Coração de Jesus, Bauru, Brasil), Cardoso MA, Ferreira SRG (Departamento de Nutrição, Saúde Pública, Universidade de São Paulo, Brasil).

\section{REFERÊNCIAS}

1. Reaven GM. Role of insulin resistance in human disease. Diabetes. 1988;37:1595-607.

2. Galassi A, Reynolds K, He J. Metabolic syndrome and risk of cardiovascular disease: a meta-analysis. Am J Med. 2006;119(10):812-9.

3. Reaven GM. Role of insulin resistance in human disease (syndrome X): and expanded definition. Annu Rev Med. 1993;44:121-31.

4. Pan XR, Li GW, Hu YH, Wang JX, Yang WY, An ZX, et al. Effects of diet and exercise in preventing NIDDM in people with impaired glucose tolerance:The Da Qing IGT and Diabetes Study. Diabetes Care. 1997;20(4):537-44.

5. Tuomilehto J, Lindström J, Eriksson JG, Valle TT, Hämäläinem H, llanne-Parikka $\mathrm{P}$, et al. Prevention of type 2 diabetes mellitus by changes in lifestyle among subjects with impaired glucose tolerance. N Engl J Med. 2001;344(18):1343-50.

6. Bray GA, Culbert IW, Champagne CM, Crow MD, Dawson L, Eberhardt B, et al. Diabetes Prevention Program Research Group. Reduction in the incidence of type 2 diabetes with lifestyle intervention or metformin. N Engl J Med. 2002;346(6):393-403.

7. Eriksson KF, Lindgärde F. Prevention of type 2 (non-insulin-dependent) diabetes mellitus by diet and physical exercise. The 6-year Malmo feasibility study. Diabetologia. 1991;34:891-989.

8. Japanese-Brazilian Diabetes Study Group. Diabetes mellitus e doenças associadas em nipo-brasileiros. Green Forest do Brasil; 2004.

9. Gimeno SGA, Ferreira SRG, Franco LJ, Hirai A, Matsumura L, Moisés RCS, et al. Prevalence and 7-year incidence of type 2 diabetes mellitus in a Japanese-Brazilian population: an alarming public health problem. Diabetologia. 2002;45(12):1635-8.

10. Ferreira SRG, Gimeno SGA, Hirais AT, Harima $H$, Matsumura L, Pittito BA. Effects of an intervention in eating habits and physical activity in Japanese-Brazilian women with a high prevalence of metabolic syndrome in Bauru, São Paulo State, Brazil. Cad Saúde Pública. 2008;24(Sup.2):S294-302.

11. Matsudo S, Timoteo A, Matsudo V, Andrade E, Oliveira LC, Bragion C. Questionário Internacional de Atividade Física (IPAQ): estudo de validade e reprodutibilidade no Brasil. Rev Bras Ativ Fis Saúde. 2001;6(2):5-18.

12. Craig $C L$, Marshall $A L$, Sjostrom $M$, Bauman $A E$, Booth $M L$, Ainsworth BE, et al. International Physical Activity Questionnaire - IPAQ: 12-country reability and validity. Med Sci Sports Exerc. 2003;35(8):1381-95.

13. Alberti G, Zimmet P. On behalf of the IDF Task Force on Epidemiology and Prevention. The IDF consensus worldwide definition of the metabolic syndrome. Disponível em: http://www.idf.org/ webdata/docs/IDF Meta def final.pdf. Acesso em: Maio 23, 2007.

14. Sartorelli DS, Sciarra EC, Franco LJ, Cardoso MA. Beneficial effects of short-term nutritional counseling at the primary health care level among Brazilian overweight adults. Public Health Nutr. 2005;8(7):820-5.

15. World Health Organization \& Food and Agricultural Organization. The scientific basis for diet, nutrition and the prevention of type 2 diabetes. Geneva; 2003.

16. Tabela Brasileira de Composição de Alimentos / Nepa, Unicamp, 2006. Disponível em: http://www.unicamp.br/nepa/taco/tabela. php. Acesso em: Set 32005.

17. Resources Council, Science and Technology Agency. Standard tables of food composition in Japan. $4^{\text {th }}$ ed. Tokyo: Women's University of Nutrition Press; 1993.

18. Tomita LY, Cardoso MA. Relação de medidas caseiras, composição química e receitas de alimentos nipo-brasileiros. São Paulo: Editora Metha; 2002.

19. WillettWC, Stampfer MJ.Total energy intake: implications for epidemiological analyses. Am J Epidemiol. 1986;124(1):17-27.

20. Margestts BM, Nelson M. Desing concepts in nutrition epidemiology. Oxford: Oxford University Press; 1998.

21. Chapman-Novakofski K, Karduck J. Improvement in knowledge, social cognitive theory variables, and movement through stages of change after a community-based Diabetes Education Program. J Am Diet Assoc. 2005;105(10):1613-6.

22. Lissner L, Heitman BL. Dietary fat and obesity: evidence from epidemiology. Eur J Clin Nutr. 1995;49:79-90.

23. Heitman BL, Lissner L, Thorkild IAS, Bengtsson C. Dietary fat intake and weight gain in women genetically predisposed for obesity. Am J Clin Nutr. 1995;61(6):1213-7.

24. Astrup A,Toubro S, Raben A, Skov A. The role of low-fat diets and fat substitutes in body weight manage-men: what have we learned from clinical studies? J Am Diet Assoc. 1997;97(suppl):S82-7.

25. Nutrition and your Health. Dietary Guidelines for Americans, 2000. 5.ed. Washington: U.S. Department of Agriculture; 2000. Disponível em: http://www.nal.usada.gov/fnic/dga/index.html. Acesso em: Set 142006.

26. Freire RD, Cardoso MA, Gimeno SGA, Ferreira SRG; for the Japanese-Brazilian Diabetes Study Group. Dietary fat is associated with metabolic syndrome in Japanese-Brazilians. Diabetes Care. 2005;28(7):1779-85

27. Liao D, Asberry PJ, Shofer JB, Callahan H, Matthys C, Boyko EJ, et al. Improvement of BMI, body composition and body fat distribution with lifestyle modification in Japanese Americans with impaired glucose tolerance. Diabetes Care. 2002;25(9):1504-10.

28. Damião R, Castro TG, Cardoso MA, Gimeno SGA, Ferreira SRG; for the Japanese-Brazilian Diabetes Study Group. Dietary intake associated with metabolic syndrome in a cohort of Japanese ancestry. Br J Nutrition. 2006;96(3):532-8.

29. Weggemans RM, Zock PL, Katan MB. Dietary cholesterol from eggs increases the ratio of total cholesterol to high-density lipoprotein cholesterol in humans: a meta analysis. Am J Clin Nutr. 2001;73(5):885-91.

30. Brown L, Rosner B, Willett WW, Sacks FM. Cholesterol-lowering effects of dietary fiber: a meta analysis. Am J Clin Nutr. 1999;69(1):30-42.

31. Kasim-Karakas SE, UImario RU, MuellerWM, Peerson J. Changes in plasma lipo-proteins during low-fat, high-carbohydrate diets: effects of energy intake. Am J Clin Nutr. 2000;71:1439-47.

32. Clarke SD. Polyunsaturated fatty acid regulation of gene transcription: a mechanis to improve energy balance and insulin resistance. Br J Nutrition. 2000;83:S59-66.

33. Harris AD, McGregor JC, Perencevich EN, Furuno JP, Zhu J, Peterson DE, et al. The use and interpretation of quasi-experimental studies in medical informatics. J Am Med Inform Assoc. $2006 ; 13(1): 16-23$ 